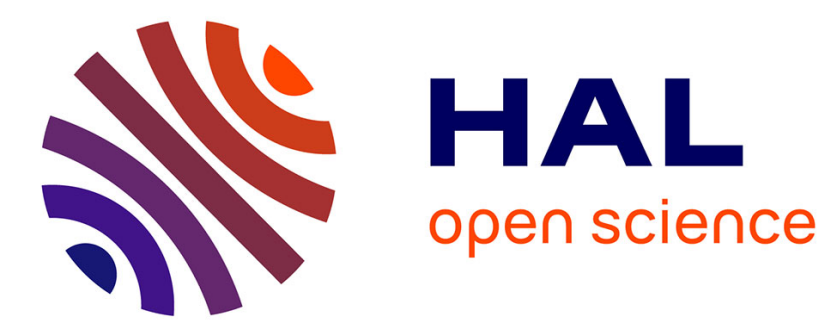

\title{
Loïc Lafargue de Grangeneuve, L'Etat face aux rave-parties. Les enjeux politiques du mouvement techno
}

Jean-Christophe Sevin

\section{- To cite this version:}

Jean-Christophe Sevin. Loïc Lafargue de Grangeneuve, L'Etat face aux rave-parties. Les enjeux politiques du mouvement techno. 2011. halshs-01933888

\section{HAL Id: halshs-01933888 \\ https://shs.hal.science/halshs-01933888}

Submitted on 24 Nov 2018

HAL is a multi-disciplinary open access archive for the deposit and dissemination of scientific research documents, whether they are published or not. The documents may come from teaching and research institutions in France or abroad, or from public or private research centers.
L'archive ouverte pluridisciplinaire HAL, est destinée au dépôt et à la diffusion de documents scientifiques de niveau recherche, publiés ou non, émanant des établissements d'enseignement et de recherche français ou étrangers, des laboratoires publics ou privés. 


\title{
Lectures
}

Les comptes rendus

I

\section{Loïc Lafargue de Grangeneuve, L'Etat face aux rave-parties. Les enjeux politiques du mouvement techno}

\author{
J EAN-CHRISTOPHE SEVIN
}

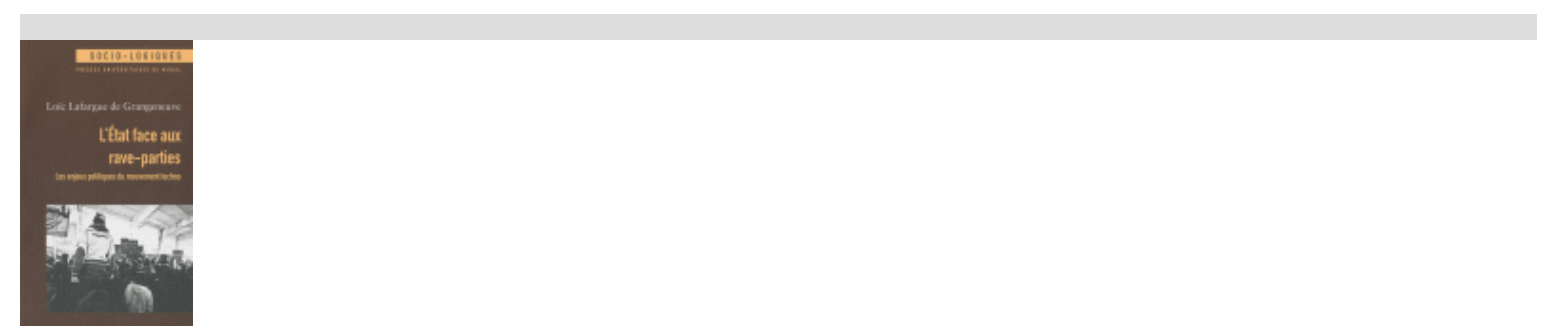

Loïc Lafargue de Grangeneuve, L'Etat face aux rave-parties. Les enjeux politiques du mouvement techno, Presses universitaires du Mirail, coll. « Socio-logiques », 2010, 170 p., EAN : 9782810701209.

Vous pouvez commander cet ouvrage sur le site de notre partenaire Decitre

\section{Texte intégral}

PDF

L'ouvrage de Loïc Lafargue de Grangeneuve, qui traite des relations entre l'Etat et les acteurs des raves-parties, est tiré d'une enquête financée par l'INHESS (Institut National des Hautes Etudes de Sécurité). Cela a permis à son auteur d'accéder aux services du Ministère de l'Intérieur et autres administrations en charge de la gestion des raves. Il a ainsi recueilli des données très riches qu'il traite avec les outils d'analyse de la sociologie des politiques publiques. De ce fait, il apporte un éclairage bienvenu sur ce phénomène, notamment dans l'analyse de la série de changements et de réajustements que l’Etat a opérés dans sa gestion des free-parties en raison des conséquences imprévues des politiques engagées ${ }^{1}$. Dans cette perspective l'importance des free-parties (raves sauvages, non autorisées), que connaît la France dans la seconde moitié des années 1990, est considérée comme un effet pervers de la politique d'interdiction des raves sous toutes ses formes dans cette période. Car cela favorise le renforcement des raves clandestines au détriment des raves légales qui subissent de plein fouet cette politique d'interdiction, par le fait même de leur signalement aux autorités. Alors que les raves clandestines, dès lors qu'elles restent invisibles, échappent aux interdictions. Et lorsque les forces de l'ordre les détectent, elles sont la plupart du temps non seulement prises de vitesse mais aussi démunies opérationnellement et juridiquement pour intervenir. Le rapport de force s'inverse ensuite avec l'adoption d'une nouvelle législation en 2001 (entrée en application au printemps 2002) qui soumet les rassemblements de plus de 250 personnes à un régime de déclaration préalable sous peine de saisie du matériel de sonorisation en cas d'infraction. La mesure est efficace et le nombre de raves illégales diminue fortement avec cette législation. Mais cette politique comporte un point aveugle avec la persistance de raves illégales de grande ampleur, susceptibles de provoquer des affrontements violents. Et c'est à la suite de l'un de ces rassemblements importants, le teknival du 15 août 2002, que le Ministère de l'Intérieur de Nicolas Sarkozy décide d'infléchir sa politique en faisant émerger des représentants des free-parties pour négocier avec eux la mise en place de teknivals (grandes raves pouvant durer plusieurs jours) « officiels » et co-gérés. Mais ces rassemblements de plusieurs dizaines de milliers de personnes s'avèrent problématiques en raison de leurs coûts, liés notamment à la mobilisation des différents services de l'administration, de même que leur lieu d'implantation suscite des controverses récurrentes. Cela pousse l'Etat à infléchir de nouveau sa politique à partir de 2005, en tentant de favoriser les rassemblements de taille modeste considérés comme plus faciles à gérer. Mais ces derniers, ajustés à la loi, sont rares. Les causes de cet état de fait sont à chercher d'une part, dans le cadre contraignant imposé aux organisateurs et d'autre part, dans les réticences des administrations déconcentrées et des collectivités locales face à la mauvaise réputation des raves. En cela, le régime de déclaration préalable fonctionne en pratique comme un dispositif d'interdiction. Loïc Lafargue de Grangeneuve montre que la nette diminution des rassemblements clandestins " ne s'est pas traduite pas un phénomène d'institutionnalisation des rave-parties » (p.81). Pour tenter de faire évoluer cette situation, le Ministère de l'Intérieur cherche alors à assoir l'autorité des médiateurs qu'il a mis en place dans les préfectures dès 2002. Ceux-ci étaient censés favoriser l'obtention d'autorisations pour les organisateurs de raves qui rempliraient les critères imposés par le 
cadre légal en facilitant leurs rapports avec les préfectures et les collectivités locales. Un autre volet de cet infléchissement de la politique du Ministère de l'Intérieur concerne le relèvement du seuil au-delà duquel les raves sont soumises à une déclaration préalable. En 2006, celui-ci passe de 250 à 500 personnes. Volet complémentaire note l'auteur, dans la mesure où le régime de déclaration préalable est difficile à appliquer et qu'il convient alors de le réserver aux rassemblements de taille moyenne. Ce ne sont ici que les grandes lignes d'une politique qui a ses constantes et ses revirements et que l'auteur détaille dans ses différentes implications. Appuyé par de longs extraits d'entretiens qui donnent corps au propos, le lecteur suit en détail les modalités concrètes de l'action des forces de l'ordre concernant la surveillance du territoire pour contrer l'apparition de raves sauvages jusqu'à celles concernant la gestion des flux des teknivaliers. La sociologie des politiques publiques éclaire les aspects de la gestion des raves au regard des nouvelles normes de management et de coopération des différents services de l'Etat tout comme les effets de résistances locales aux rave-parties que l'auteur décrypte comme une variante du syndrome NIMBY (not in my backyard). Sont ainsi analysées les tensions entre les services de l'Etat et les collectivités locales quand les premiers se retrouvent aux côtés des organisateurs contre les élus locaux résistant farouchement à la tenue d'une rave sur leur territoire. Mais les tensions peuvent aussi survenir à propos de la question de la drogue face à laquelle deux logiques entrent en tension, avec une démarche de répression du trafic menée par les forces de police et une politique de réduction des risques portée par le monde associatif qui cherche à informer les consommateurs considérés comme des êtres rationnels sur les conséquences de leurs actes. Cependant, si l'analyse de la politique de l'Etat envers les rave-parties est convaincante, celle qui concerne la politique des rave-parties l'est moins. L'auteur semble accorder beaucoup plus d'importance, dans l'exposition des motivations du mouvement des free-parties, au « positionnement rebelle » ou aux attitudes d'évitement des autorités qu'aux valeurs souvent revendiquées comme l'amateurisme ou la gratuité. Si elles ont pu être dévoyées, c'est néanmoins celles-ci que les sound-systems ont réussi à imposer à l'Etat avec l'organisation des teknivals officiels. Même si la fête libre se voyait lourdement encadrée, encourageant la fracture avec ceux qui refusaient tout compromis comme cela est décrit par ailleurs. Enfin, la mise sur le même plan de la marginalité sociale et des minorités ethniques ou sexuelles pour accréditer l'idée d'un « lien récurrent entre techno et marginalité sociale » (p.29) est discutable 2 . De plus, catégorisé de cette manière, la gestion quasi-exclusive d'un phénomène culturel et artistique par le Ministère de l'Intérieur et non par le Ministère de la Culture, qui apparait à l'auteur comme un paradoxe, n'en est pas vraiment un. Ne serait-ce pas plutôt une manifestation parmi d'autres d'une dérive sécuritaire que l'on observe depuis plusieurs années?

\section{Notes}

1 L'auteur fait ici référence à Patrice Duran (Penser l'action publique, Paris, LGDJ, 1999) pour qui « la mise au jour des conséquences non-attendues et non-voulues de l'action se révèle fondamentale pour une sociologie de l'action publique compte tenu du fait que les autorités publiques doivent en assumer la responsabilité ». (Cité p.47)

2 Alors même que l'auteur note ailleurs que de nombreux raveurs sont étudiants

\section{Pour citer cet article}

Référence électronique

Jean-Christophe Sevin, « Loïc Lafargue de Grangeneuve, L'Etat face aux rave-parties. Les enjeux politiques du mouvement techno », Lectures [En ligne], Les comptes rendus, 2011, mis en ligne le 12 février 2011, consulté le 24 novembre 2018. URL : http://journals.openedition.org/lectures/1273

\section{Rédacteur}

Jean-Christophe Sevin

Sociologue. Chercheur associé au Centre Norbert Elias (EHESS - Marseille).

Articles du même rédacteur

Jussi Parikka, Qu'est-ce que l'archéologie des média [Texte intégral]

Jean-Pierre Cometti, Conserver/Restaurer. L'œuvre d'art à l'ère de sa préservation technique [Texte intégral]

Lello Savonardo, Sociologie de la musique. La construction sociale du son des « tribus » au numérique [Texte intégral] Tous les textes

\section{Droits d'auteur}

(c) Lectures - Toute reproduction interdite sans autorisation explicite de la rédaction / Any replication is submitted to the authorization of the editors

Drag to outliner or Upload

Close 